\title{
Modelo de Programación Lineal Multiobjetivo para la Logística Inversa en el Sector Plástico de Polipropileno
}

\author{
Efraín De la Hoz ${ }^{(1)}$, Jorge Vélez ${ }^{(2)}$ y Ludys López ${ }^{(3)}$ \\ (1) Universidad de Cartagena, Facultad de Ciencias Económicas, Programa de Administración Industrial. \\ Campus Piedra de Bolívar, Cartagena, Colombia. (e-mail: edelahozg@unicartagena.edu.co). \\ (2) Polipropileno del Caribe S.A., Gerencia de Investigación y Desarrollo, Mamonal Km 8, Cartagena, \\ Colombia. (e-mail: jorge.velez@esenttia.co). \\ (3) Universidad Simón Bolívar, Facultad de Ingeniería, Programa de Ingeniería Industrial. Carrera 59 No. \\ 59-65 Sede 3 bloque B 5 piso, Barranquilla, Colombia. (e-mail: Iulopez@unisimonbolivar.edu.co).
}

${ }^{*}$ Autor a quien debe ser enviada la correspondencia

Recibido Abr. 20, 2017; Aceptado Jun. 7, 2017; Versión final Jul. 5, 2017, Publicado Oct. 2017

\begin{abstract}
Resumen
En esta investigación se presenta un modelo de programación lineal multiobjetivo para la logística inversa del sector plástico de polipropileno. Para ello, se realizó una revisión de los referentes teóricos relacionados con el proceso de recuperación del plástico de polipropileno a partir de residuos sólidos urbanos y los desperdicios de los procesos industriales. Seguidamente se definió un modelo de programación lineal multiobjetivo el cual incorpora las variables y parámetros identificados en el diseño del proceso de logística inversa. Finalmente se hace un análisis comparativo de un caso de estudio para determinar el impacto del modelo en los resultados del programa de planeación logística. Se muestra un mejoramiento del $12,6 \%$ en los costos asociados al programa de planeación. Al mismo tiempo se optimiza el Índice de Degradación a la Procesabilidad lo que genera una buena calidad del polipropileno reciclado.
\end{abstract}

Palabras clave: logística inversa; índice de degradación; residuos sólidos; plástico; programación lineal multiobjetivo

\section{Multiobjective Linear Programming Model for Reverse Logistics in the Polypropylene Plastics Industry}

\begin{abstract}
A model of multi-objective linear programming for reverse logistics sector of plastic polypropylene is presented in this paper. For this, a review of the theoretical framework related to the recovery process of polypropylene plastic from municipal solid waste and waste of industrial processes was done. Next, a multiobjective linear programming model which incorporates the variables and parameters identified in the design of the reverse logistics process was defined. Finally, a comparative analysis of a case study is done to determine the impact of the model on the results of the program logistics planning. An improvement of $12.6 \%$ was found in the costs associated with the program planning. At the same time, the Degradation to Processability Index is optimized which generates good quality recycled polypropylene.
\end{abstract}

Keywords: reverse logistics; degradation index; solid waste; plastic; linear multiobjective programming 


\section{INTRODUCCIÓN}

Con la evolución de la industria, la gestión de residuos se he convertido en un problema cada vez de mayores dimensiones que demanda la toma de decisiones gerenciales a nivel operativo y estratégico (Rubio, 2003). De esta manera, autores como Marciel et al., (2016) proponen sistemas de gestión de residuos sólidos que permitan planificar y controlar su reutilización o eliminación. Así mismo, paralelamente se ha generado una tendencia de separación en la fuentes urbanas de residuos sólidos como parte de los procesos de reciclaje (Poletto y da Silva, 2009). En contraste con lo anterior, los esfuerzos para desarrollar modelos que integren las dimensiones económicas y medioambientales en la gestión de la cadena de suministros inversa son escasos; con lo cual, la modelación en procesos logísticos de reciclaje toma importancia estratégica en la sostenibilidad de las cadenas de suministro (Feitó et al., 2016). En este sentido, la investigación de operaciones, se constituye en una herramienta científica valiosa para soportar la toma de decisiones en la asignación de recursos escasos, utilizando para tal fin modelos matemáticos de optimización que representan una situación real (Winston, 2005), desde las cuales establecer estrategias y lineamientos que generen efectos positivos para su competitividad (Mercado et al., 2011).

En general, una Cadena de Suministro (CS) puede definirse como una serie de procesos y flujos que se realizan dentro y fuera de las industrias y en diferentes etapas que se intercambian para satisfacer las necesidades de los clientes internos y externos (Chopra y Meindl, 2008). Ballou (2004) establece la CS como el conjunto de actividades a lo largo de un proceso en el que se agrega valor a las materias primas para convertirlas en productos terminados. De lo anterior, dada la naturaleza compleja y dinámica de las CS, se hace necesario diseñar modelos que permitan analizar su comportamiento a partir del cual definir o mejorar nuevos sistemas para su funcionamiento (Sanchis et al., 2009).

Por otra parte, la logística inversa se entiende como el desarrollo de procesos de recolección, desmonte y desmembramiento de productos o materiales usados con objetivo de maximizar su aprovechamiento o uso sostenible y en últimas su destrucción (Cabeza, 2012). Así mismo, Reverse Logistics Executive Council define la logística inversa como el proceso mediante el cual se planea, implementa y controla el costo del flujo efectivo de materias primas, inventario de productos en proceso y terminados, así como el flujo de información desde el punto de consumo al punto de origen, con el propósito de reducir el impacto ambiental y/o recuperar valor (Sbihi y Eglese, 2010). La logística inversa de los plásticos incluido el polipropileno, en general es el proceso mediante el cual el plástico post-consumo provenientes de los rellenos sanitarios o basureros (Residuos Sólidos Urbanos) o el industrial provenientes de las empresas fabricantes y transformadoras de plásticos, son recuperados permitiendo su posterior utilización. Este proceso se clasifica en diferentes etapas en la cadena de logística inversa. En la figura 1 se muestra de manera esquemática el flujograma del proceso de recuperación (logística inversa) del plástico de polipropileno para su disposición en la producción industrial.

En la etapa 1 (Recolección y separación manual) es el proceso en el cual se recoge manualmente los plásticos de los residuos sólidos urbanos y se clasifica por tipo de plástico y el nivel de contaminación con otros materiales, el cual se comercializa por unidad de peso y su valor comercial es variable y acorde y su nivel de clasificación. Seguidamente, el plástico es transportado al punto de acopio donde es picado o troceado en tamaño que oscila de 10 a $50 \mathrm{~mm}$ de lado, empacado y almacenado (Al-Salem et al., 2009). En la etapa 2 (Separación mecánica de los plásticos) se clasifica el plástico mediante técnicas de flotación y hundimiento por diferencia de densidad (Al-Salem et al., 2009), utilizando una piscina llena con una solución de densidad conocida, que normalmente es agua mezclada con cristales de sal, para así obtener la densidad de la solución requerida. El plástico, que tiene una densidad que lo caracteriza, flota a diferentes niveles en la solución dependiendo del tipo de plástico, facilitando así su separación por medio de mallas separadoras. A continuación el plástico, es conducido al proceso de lavado con una solución ácida o básica, pasando posteriormente a ser enjuagado con agua, secado y almacenado pasando posteriormente a la etapa de molienda. El polipropileno proveniente de las plantas de producción que llega libre de contaminante de otros materiales, entran al proceso de lavado y molienda, previo troceado o picado.

En la etapa 3 (Proceso de molienda del polipropileno) el proceso de molienda reduce el tamaño del plástico al tamaño según los requerimientos de la máquina del proceso siguiente (proceso de extrusión). Existen diversos tipos de tecnología según el tamaño al cual se quiera llegar, en general puede llegarse a obtener hojuelas de 2 a $5 \mathrm{~mm}$ o finalmente polvo, según el tipo de molino del que se disponga (Al-Salem et al., 2009). Una vez extraído el polipropileno del molino pasa al proceso siguiente, o empacado y almacenado para ser comercializado posteriormente. En la etapa 4 (Proceso de Extrusión y Aditivación del Polipropileno) el material pasa a la zona de alimento de la máquina extrusora, cae al cilindro principal (cañón) donde se encuentra con el husillo (tornillo de Arquímedes) calentado por resistencias eléctricas a temperatura de $220^{\circ} \mathrm{C}$ aproximadamente para fundir el polipropileno a procesar (Al-Salem et al., 2009). Este proceso consiste en el prensado y moldeado del material procesado anteriormente hasta obtener la forma usual de 
cilindro fino y alargado. En la Aditivación como mejora de la degradación, el nivel de la degradación del plástico de polipropileno reciclado es influenciado por su historia de origen y también por el sistema base de estabilización particular utilizado (Hamskog et al, 2004). De esta manera, la adición de un estabilizador o antioxidante en concentraciones que depende del grado de degradación del material antes de ser procesado, es un método eficaz para la actualización o bloqueo de la degradación del polipropileno por ello se requiere conocer el nivel de degradación antes y después de ser extruido el material a través de técnicas de ensayo en laboratorio (Tochácek y Jancar, 2012).

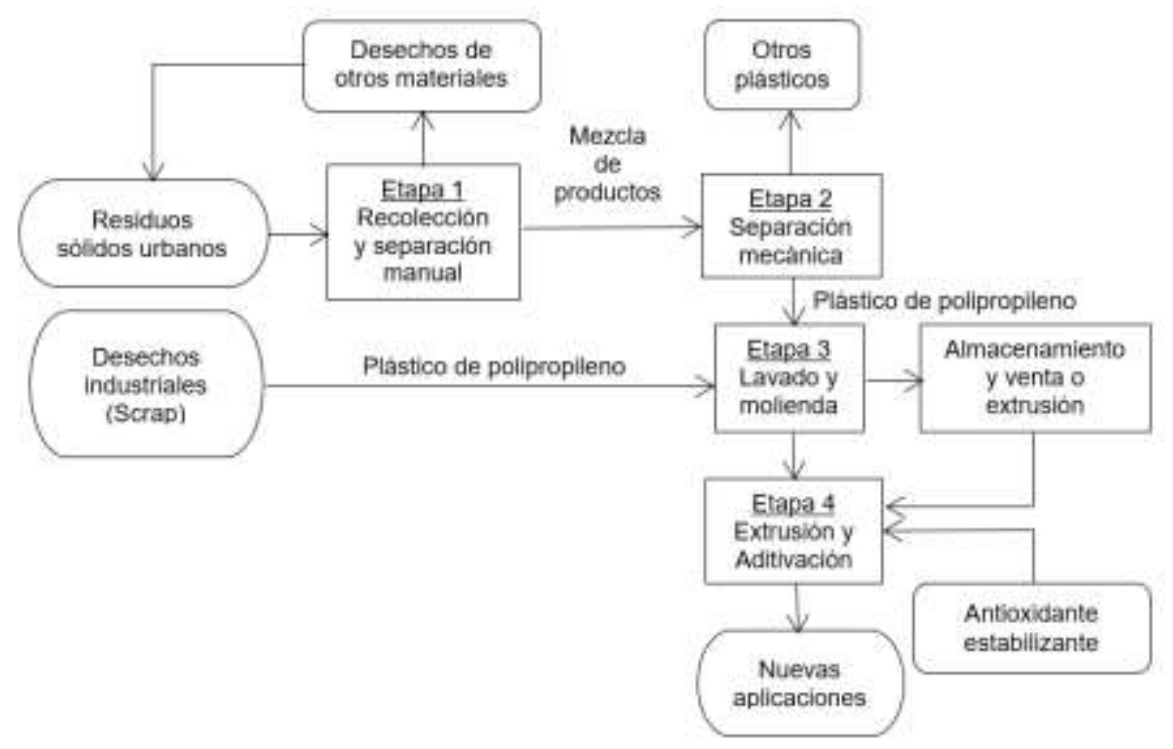

Fig. 1: Flujograma de la logística inversa del Plástico de Polipropileno

Según Fontalvo et al., (2010) para emprender acciones de mejora, es fundamental el análisis y descripción de sus procesos para identificar debilidades hacia las cuales direccionaras. En este sentido, en los procesos involucrados para la logística inversa de los plásticos desde los rellenos sanitarios hasta su disponibilidad en la producción industrial, la variable con mayor impacto en la toma de decisiones corresponde al costo del producto final (Gent et al, 2010), sin embargo otra variable no menos importante es la estabilidad de la degradación del polipropileno recuperado como factor determinante de la calidad del polipropileno reciclado, es decir, recuperar un material plástico de manera que quede apto para nuevamente ser procesado (Tochácek y Jancar, 2012). Por lo anterior, es importante que se optimicen los costos de producción y el índice de degradación para cumplir con las especificaciones de calidad del cliente (Vélez y De la Hoz, 2016).

En esta investigación, se presenta un modelo de PLM para la logística inversa del sector plástico de polipropileno, en el que se hace una análisis para optimizar los factores relevantes que influyen a lo largo de la logística inversa de toda la cadena del proceso, implementándose en un caso de estudio en una empresa de la zona industrial Mamonal de la ciudad de Cartagena dedicada al procesamiento y comercialización de plásticos reciclados incluyendo el polipropileno, el cual es importante para el mejoramiento competitivo de las organizaciones empresariales en la medida que se optimiza el proceso de toma de decisiones.

\section{METODOLOGÍA}

La presente investigación se enmarca en un análisis cuantitativo y descriptivo de un modelo de programación lineal multiobjetivo para la logística inversa del sector plástico de polipropileno para lo anterior se definieron los proceso asociados a la recuperación del plástico de polipropileno proveniente de los residuos industriales y de los residuos sólidos urbanos, lo que permitió identificar las variables y parámetros claves. Seguidamente se establecieron dos funciones objetivos, Costo de la logística inversa de polipropileno $\left(Z_{1}\right)$ y Degradación del polipropileno reciclado $\left(Z_{2}\right)$, con las restricciones identificadas en el proceso y se valida el modelo analizando el mejoramiento de los costos y el índice de degradación asociados al programa de planeación en un horizonte de seis meses en una empresa del sector plástico en la ciudad de Cartagena a partir de la solución dada por el modelo. Para el caso de la función objetivo $Z_{1}$, con $\mathrm{P}$ periodos de producción, $\mathrm{N}$ centros de acopio y $\mathrm{R}$ posibles rutas de transporte, la función lineal se planteó teniendo en cuenta los costos de transporte y almacenamiento en el periodo $p$ (primer paréntesis), costos de material de producto fabricado (segundo paréntesis), costos de la fuerza de trabajo en horas hombre(tercer y cuarto paréntesis), los costos causados por las contrataciones y despidos (quinto y sexto paréntesis), Costo de horas ociosas (séptimo paréntesis), costo inventario promedio en bodegas (octavo paréntesis), costo pedidos satisfechos y no satisfechos (noveno y décimo paréntesis) y costo de aditivo (undécimo paréntesis). La expresión matemática se muestra en la Ecuación 1. 


$$
\begin{aligned}
\operatorname{Min} Z_{1}= & \sum_{p=1}^{P}\left[\left(\sum_{n=1}^{N} \sum_{r=1}^{R} \operatorname{Trans}_{r p} * \operatorname{CTrans}_{r p}+\operatorname{Alm}_{n p} * C A l m\right)+\left(P d_{p} * C M P\right)+\left(H h R_{p} * C H h R\right)+\left(H E_{p} * C H E\right)+\left(C_{p} * P C_{p}\right)+\right. \\
& \left.\left(D_{p} * P D_{p}\right)+\left(H h O_{p} * C H O\right)+C I n v *\left(\frac{\operatorname{Inv}_{p}+I n v_{p-1}}{2}\right)+\left(P S_{p} * C P S\right)+\left(P N S_{p} * C P N S\right)+\left(A d t_{p} * C A d t\right)\right]
\end{aligned}
$$

Donde $p$ son los periodos de producción $(p=1 \ldots P), n$ los centros de acopio $(n=1, \ldots, N), r$ la ruta del transporte $(r=1, \ldots, R)$, Trans $r p$ es transporte por la ruta $r$ hasta en el periodo $p, C_{\text {Trans }}$ es el costo del transporte por la ruta $r$ hasta en el periodo $p, A / m_{n p}$ el almacenaje en el centro de acopio $n$ en el periodo $p$, $C A / m$ el costo de almacenaje, $P d_{p}$ la producción realizada en el periodo $p, C M P$ el costo de materia prima, $H h R_{p}$ horas hombre regular en el periodo $\mathrm{p}, \mathrm{CH} h \mathrm{R}$ : Costo hora hombre regular, $H E p$ horas extras en el periodo $\mathrm{p}, \mathrm{CHE}$ costo horas extras, $C_{p}$ contrataciones en el periodo $\mathrm{p}, P C_{p}$ costo del perjuicio por las contrataciones en el periodo $\mathrm{p}, D_{p}$ despidos en el periodo $\mathrm{p}, P D_{p}$ costo del perjuicio por los despidos en el periodo $\mathrm{p}$, HhOp horas hombre ociosas en el periodo $\mathrm{p}, \mathrm{CHO}$ costo hora hombre ociosa, Inv $v_{p}$ inventario en bodegas en el periodo $\mathrm{p}, \mathrm{CInv}$ costo por inventario en bodegas, $P N S_{p}$ pedidos no satisfechos en el periodo p, CPNS costo de los pedidos no satisfechos, $P S_{p}$ producción subcontratadas en el periodo p, CPS costo por producción subcontratadas, $A d t_{p}$ aditivo usado en el periodo $\mathrm{p}, C A d t$ costo aditivos. Así mismo en la investigación experimentalmente se determinó el comportamiento lineal del índice de degradación a la procesabilidad en función de la concentración de aditivo estabilizante adicionada en el periodo $p$ mediante un análisis de regresión simple, en el que obtuvo un R-cuadrada del $98.48677 \%$. (Ec. 2 ), donde $P D I_{0}$ es el Índice de Degradación a la Procesabilidad al inicio del proceso de reciclaje, $m$ la pendiente de la línea obtenida en la relación del PDI y el Adt, $A d t_{p}$ la concentración del aditivo estabilizante adicionada en el periodo $\mathrm{p}$.

$\operatorname{Min} Z_{2}=\sum_{p=1}^{P}\left(P D I_{p}+m * A d t_{p}\right)$

Seguidamente se plantean las restricciones de producción (Ec. 3), de mano de obra u horas hombre (Ec. 4), de contrataciones despidos (Ec. 5), de horas extras y ociosas de los trabajadores (Ec. 6), de inventarios en las bodegas (Ec. 7), de producción no entregada a tiempo (perjuicio insatisfacción por los retrasos (Ec. 8), por producción subcontratada en empresa externa (Ec. 9), por concentración de aditivo estabilizante agregado (Ec. 10) y de no negatividad (Ec. 11). En la ecuación 10, se tuvo en cuenta los resultados para minimizar el Índice de Degradación a la Procesabilidad planteado por Vélez y De la Hoz (2016).

\begin{tabular}{|l|r|}
\hline$\left(D m_{p}+\operatorname{Inv} v_{p}+\operatorname{Inv} v_{p-1}\right) \leq\left(P d_{p}\right) \leq\left(C p d_{p}\right)$ & $(3)$ \\
\hline$H h_{p} \leq M H h$ & $(4)$ \\
\hline$C_{p} \leq M C ; \quad D_{p} \leq M D$ & $(5)$ \\
\hline$H E_{p} \leq X \% * H h R ; \quad H h O_{p} \leq H h R_{p}$ & $(6)$ \\
\hline$I n v_{p} \leq M I n v$ & $(7)$ \\
\hline$P N S_{p} \leq D m_{p}$ & $(8)$ \\
\hline$P S_{p} \leq C P S$ & $(9)$ \\
\hline $0,05 \% \leq A d t_{p} \leq 0,6 \%$ & $(10)$ \\
\hline$I n v_{p}, H h R_{p}, C_{p}, D_{p}, H E_{p}, H h O_{p}, \operatorname{Inv}_{p}, P N S_{p}, P S_{p}, A d t_{p} \geq 0$ & $(11)$ \\
\hline
\end{tabular}

En estas ecuaciones, $p=1 \ldots P, H h O_{p}$ las horas ociosas de los trabajadores en el periodo $\mathrm{p}$, el número máximo de trabajadores establecidos por política por la empresa, $M C$ número máximo contrataciones de trabajadores establecidos por políticas de la empresa, MD máximo despidos de trabajadores establecidos por políticas de la empresa, $X \% H R$ porcentaje máximo de horas extras establecidos por ley, MInv máximo inventario permitido ya sea por capacidad o por política, $D m_{p}$ demanda en el periodo, $P D l_{o p}$ indice de degradación a la procesabilidad del polipropileno a reciclar al inicio del periodo $\mathrm{p}$, Cpd capacidad de la producción. Para la validación del modelo de PLM, se utilizó la información del programa de producción (mayo- octubre 2016) de una empresa dedicada al procesamiento y comercialización de plásticos reciclados.

\section{RESULTADOS Y DISCUSIÓN}

El análisis de la literatura, se evidenció las importancia de las herramientas matemáticas para permitan diseñar CS dedicadas a la recuperación de materiales reciclables. La recuperación de materiales que comparten recursos, unido a las condiciones de incertidumbre tanto en la generación como en la demanda, constituyen un reto para el diseño y gestión de la CS. De esta manera, existe el compromiso de brindar desarrollar procesos que aporten al desarrollo sostenible, a la vez que se cumplen con objetivos económicos, sociales y ecológicos en estos diseños (Feitó et al., 2016). El modelo propuesto optimiza dos objetivos clave para el proceso objeto de estudio, por lo que se considera pertinente y original, a la vez que complementa otros 
modelos existentes en la literatura científica (Chen et al., 2007; Gou et al., 2008 y Lee y Dong, 2009). La propuesta del artículo soluciona parte de la contradicción existente entre la necesidad del proceso de optimizar estos objetivos y la ausencia, al menos de forma explícita, de una herramienta metodológica que lo permita, por tal razón se considera que constituye una contribución al conocimiento científico.

Con el propósito de validar el modelo y evidenciar el mejoramiento en los resultados, se aplicó a una empresa del sector plástico de la ciudad de Cartagena (Colombia). Específicamente se simuló un programa de producción de seis meses ejecutado en la empresa (programa actual). Los resultados del modelo (programa propuesto) se compararon con los del programa actual. En las Tablas 1 y 2, se muestra la información de demanda, tiempo laborado, aditivo estabilizante y costos utilizados. La Tabla 3 muestra los resultados entregados por el programa de producción actual y el programa propuesto obtenidos a partir de la simulación del modelo en la herramienta Solver de Excel.

Tabla 1: Información de la demanda en el periodo

\begin{tabular}{|l|c|c|c|c|c|c|}
\hline \multicolumn{1}{|c|}{ Detalle } & May & Jun & Jul & Agt & Sept & Oct \\
\hline Días Laborados. & 25 & 24 & 24 & 25 & 25 & 26 \\
\hline Horas Labordas. & 200 & 192 & 192 & 200 & 200 & 208 \\
\hline Demanda (Tn) & 30 & 30 & 25 & 30 & 30 & 27 \\
\hline Aditivo Estab. (1\%) (Tn) & 0,030 & 0,030 & 0,025 & 0,030 & 0,030 & 0,027 \\
\hline
\end{tabular}

Tabla 2: Información de costos

\begin{tabular}{|l|r|}
\hline \multicolumn{1}{|c|}{ Detalle } & \multicolumn{1}{c|}{ Valor } \\
\hline Costo de un Trab. (Incluy. Prest) & $\$ 919.620 / \mathrm{Mes}$ \\
\hline Costo Directos & $\$ 825.000 / \mathrm{Tn}$ \\
\hline Costo Aditivo Estabilizante & $\$ 10.608 .000 / \mathrm{Tn}$ \\
\hline Costo por Mantener Inventarios & $\$ 10.000 / \mathrm{Tn} / \mathrm{mes}$ \\
\hline Costo Marginal del Desabasto & $\$ 9.000 / \mathrm{Tn}$ \\
\hline Costo de Contratación & $\$ 7.000 / \mathrm{Trab}$ \\
\hline Costo de Despidos & $\$ 3.000 / \mathrm{Trab}$ \\
\hline Costo del Tiempo Regular & $\$ 3.125 / \mathrm{hr}$ \\
\hline Costo del Tiempo Extra & $\$ 3.906,25 / \mathrm{hr}$ \\
\hline Capacidad Máxima del Proceso & $0,17 \mathrm{Tn} / \mathrm{hr}$ \\
\hline Inventario Inicial & $7 / \mathrm{Tn}$ \\
\hline Inventario mínimo requerido (stock) & $10 / \mathrm{Tn}$ \\
\hline Número de operarios & 6 \\
\hline
\end{tabular}

Tabla 3: Resultados de los programas de producción actual y propuesta

\begin{tabular}{|l|r|r|}
\hline \multicolumn{1}{|c|}{ Detalle } & Programa actual & Programa propuesto \\
\hline Días laborados & 149 & 149 \\
\hline Horas laboradas & 1192 & 1192 \\
\hline Demanda requerida $(\mathrm{Tn})$ & 209 & 209 \\
\hline Aditivo estabilizante consumido $(\mathrm{Tn})$ & 2,09 & 0,491 \\
\hline Horas extras realizadas & 273,3 & 250,9 \\
\hline Costo de materia prima & $\$ 174.900 .000$ & $\$ 174.900 .000$ \\
\hline Costo horas hombre regular & $\$ 25.826 .798$ & $\$ 25.826 .798$ \\
\hline Costo horas extras & $\$ 7.854 .130$ & $\$ 3.553 .385$ \\
\hline Costo horas hombre ociosas & --- & $\$ 823.059$ \\
\hline Costo de Inventario & --- & $\$ 543.000$ \\
\hline Costo aditivo estabilizante & $\$ 26.424 .528$ & $\$ 1.124 .448$ \\
\hline Total: & $\$ 235.006 .456$ & $\$ 205.405 .631$ \\
\hline
\end{tabular}

Los resultados muestran reducción del $12,6 \%$ en el costo total del programa propuesto, siendo el costo de aditivo estabilizante el factor con mayor impacto en el ahorro, producto de la implementación de la segunda función objetivo en el modelo. Así mismo, los resultados muestran un ahorro en el costo asociado a horas extras asociada a la primera función objetivo. 


\section{CONCLUSIONES}

Del análisis y discusión de los resultados, se puede concluir lo siguiente sobre el modelo de PLM para la toma de decisiones en la planeación del proceso logístico de reciclaje del plástico de polipropileno: 1) El modelo mostró efectividad para mejorar la toma de decisiones en la planeación de programas de producción de la logística inversa del sector platico; 2) El modelo planteado logro minimizar el índice de degradación y los costos en el proceso de toma de decisiones para la planeación del programa de producción; 3) Se evidencia la importancia del modelo y su impacto en el mejoramiento de los procesos logísticos para la recuperación del plástico de polipropileno para su uso en la industria.

\section{REFERENCIAS}

Al-Salem, S., Lettieri, P. y Baeyens, J. Recycling y recovery routes of plastic solid waste (PSW): A review, Waste Management, (29), 2625-2643 (2009)

Ballou, R. Business Logistics Management. 5a Ed., Pearson Educación, México D.F., México (2004)

Cabeza, D. Logística inversa en la gestión de la cadena de suministro, Marge Books, Barcelona, España (2012)

Chen, H., Chou, H. y Chiu, Y. On the modeling and solution algorithm for the reverse logistics recycling flow equilibrium problem, Transportation Research Part C, 15(4), 218-234 (2007)

Chopra, S. y Meindl, P. Administración de la cadena de suministro, $3^{a}$ Ed., Pearson Educación, México D.F, México (2008)

Correa, A. y Gómez, R. Tecnología de la Información en la Cadena de Suministro, Rev. DYNA, (76), 37-48 (2008)

Feitó, M., Cespón, R. y Rubio, M. Modelos de optimización para el diseño de cadenas de suministros de reciclaje de múltiples productos, Ingeniare, Revista Chilena de Ingeniería, 24(1), 135-148 (2016)

Fontalvo, T., De la Hoz, E., y Cardona, D. Diseño de un plan de mejoramiento para la cadena de suministro de la empresa Drolitoral SA aplicando el Modelo SCOR, Revista Soluciones de Postgrado EIA, 1(6), 33- 53 (2010)

Gent, R., Menendez, M. y otros dos autores, Optimization of the recovery of plastics for recycling by density media separation cyclones, Resources, Conservation \& Recycling, (55), 473 (2010)

Gou, Q., Liang, L. y otros dos autores. A joint inventory model for an open-loop reverse supply chain, International Journal of Production Economics, 116(1), 28-42 (2008)

Hamskog, M., Klügel, M. y otros tres autores. The effect of base stabilization on the recyclability of polypropylene as studied by multi-cell imaging chemiluminescence y microcalorimetry, Polymer degration and stability, 86(3), 557-566 (2004)

Lee, D. y Dong, M. Dynamic network design for reverse logistics operations under uncertainty, Transportation Research Part E, 45(1), 61-71 (2009)

Marciel, T., Stumpf, M. y Kern, A. Management system proposal for planning and controlling construction waste, Revista Ingeniería de Construcción, 31(2), 105-116 (2016)

Mercado, H., Fontalvo, T. y De la Hoz, E. Análisis comparativo entre las cadenas productivas del sector textil-confecciones de la provincia de Jiangsu-China y el departamento del Atlántico-Colombia, Ingeniare, Revista Chilena de Ingeniería, 19(3), 429- 441 (2011)

Poletto, J. y da Silva, C. Influencia de la separación de residuos sólidos urbanos par reciclaje en el proceso de incineración con generación de energía, Información Tecnológica, 20(2), 105-112 (2009)

Rubio, S. El sistema de logística inversa en la empresa: análisis y aplicaciones, Tesis Doctoral, Universidad de Extremadura, Departamento de economía aplicada y organización de empresas, España (2003)

Sanchis, R., Poler, R., y Ortiz, A. Técnicas para el Modelado de Procesos de Negocio en Cadenas de Suministro, Información Tecnológica, 20(2), 29- 40 (2009)

Sbihi, A. y Eglese, R. Combinatorial optimization and Green Logistics, Annals of Operations Research, 175(1), 159-1 75 (2010)

Tochácek, J. y Jancar, J. Processing degradation index (PDI) - A quantitative measure of processing stability of polypropylene, Polymer Testing, (31), 1116-1117 (2012)

Vélez, J. y De la Hoz, E. Modelo lineal para estimar el índice de degradación a la procesabilidad del polipropileno reciclado debida a la adición de estabilizante, Ingeniare. Revista Chilena de Ingeniería, 24(1), 85-93 (2016)

Winston, W. Investigación de operaciones. Aplicaciones y algoritmos, 4a Ed-, Thomson editores S.A., México D.F., México (2005) 